\title{
SIMULATIONS OF THE 3D ISING MODEL AT CRITICALITY
}

\author{
H.W.J. BLöte AND G. KamieniaRz* \\ Laboratorium voor Technische Natuurkunde, Technische Universiteit Delft \\ P.O. Box 5046, 2600 GA Delft, The Netherlands
}

\begin{abstract}
We present some new finite-size results for the Binder cumulant that we obtained by means of a transfer-matrix perturbation expansion and by the cluster Monte Carlo methods - the Swendsen-Wang and Wolff algorithms and the largest-cluster method. A finite-size scaling analysis taking into account a correction to scaling locates the critical coupling at $K_{\mathrm{c}}=0.221649(4)$.
\end{abstract}

PACS numbers: $05.50 .+q, 64.60 .-\mathrm{i}, 64.60 . \mathrm{Fr}, 75.40 . \mathrm{Mg}, 64.60 . \mathrm{Ak}$

The three-dimensional Ising model continues to be a subject of investigations, in particular its critical behaviour. Whereas the most accurate results traditionally came from series expansions (see e.g. Refs. $[1,2]$ and references therein) and from the $\varepsilon$-expansion [3], the error margins quoted in Monte Carlo based approaches are shrinking considerably [4-6]. Due to the availability of fast computers, and the invention of ingenious cluster algorithms by Swendsen and Wang [7] and Wolff [8], such a statistical accuracy is now becoming a vailable that the results are comparable with series and $\varepsilon$-expansions. However, the numerical results of such simulations are not always in satisfactory agreement with one another. For instance, the critical points of the simple cubic Ising model as found by Baillie et al. [6] $\left(K_{\mathrm{c}}=0.221652\right.$

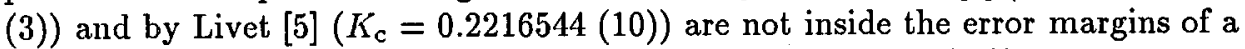
recent result by Ferrenberg and Landau [4] $\left(K_{c}=0.2216595(26)\right)$. Furthermore, the critical exponent $\nu$ obtained by Baillie et al. does not agree satisfactorily with others. Although these differences are not large, they invite the consideration of possible sources of systematic errors. In our own analysis, we made efforts to avoid, where possible, the following ones:

1. Effects due to a poor random generator;

2. Effects introduced by histogramming methods;

3. The use of a single row of pseudo-random numbers for a number of parallel simulations;

"Permanent address: Institute of Physics, A. Mickiewicz University, Poznan, Poland; present address: Institute of Theoretical Physics, University of Leuven, Celestijnenlaan 200D, Leuven, Belgium. 
4. Neglecting corrections to scaling in fitting procedures of simulation results.

We have simulated simple cubic Ising lattices with periodic boundary conditions and analyzed the amplitude ratio

$$
g_{L}=\left\langle m^{4}\right\rangle_{L} /\left\langle m^{2}\right\rangle_{L}^{2}
$$

where $m$ is the magnetization and $L-$ the linear system size. The Monte Carlo data were mainly generated using the Swendsen-Wang cluster method for $K=0.221653$. The coupling strength $K=0.221656$ used in [5] is slightly different, therefore we used finite-size scaling to correct for the linear dependence of $g_{L}$ in terms of field derivatives of the free energy.

As a consistency test, we have computed $g_{L}$ independently for $3^{3}$ and $4^{3}$ systems with periodic boundaries. Exact numerical calculation of $g_{L}$ for the smaller system hardly poses any problems, but the $4^{3}$ system is already too time-consuming unless treated carefully. A way to tackle this problem is to write the partition sum as $Z=\operatorname{Tr} T^{4}$, where $\boldsymbol{T}$ is the $65536 \times 65536$ transfer matrix, to expand $\boldsymbol{T}$ in powers of the field $h$, and to keep track of powers of $h$ during the matrix multiplications. Thus one obtains the second and fourth derivatives of $Z$ with respect to $h$, in which $g_{L}$ can be expressed. It is fortunate that the multiplications can be performed using a sparse-matrix decomposition, and that out of the 65536 terms in the trace only 433 are independent. The rest follows by the 16 -fold translation symmetry, the 4 -fold rotation symmetry, the spatial inversion symmetry and the spin-inversion symmetry of a periodic $4 \times 4$ square of the Ising spins. (For more details of the calculations, see Ref. [9].) These results for $L=3$ and $L=4$ are included in Table, and are in satisfactory agreement with our Monte Carlo results. For $L=4$, the difference with Ref. [5] is 1.8 standard deviations and 2.4 standard deviations according to our determination of the statistical error for a run of the quoted number of Swendsen-Wang steps $\left(5 \times 10^{6}\right)$. For this determination we divided runs of approximately that length in 1000 partial results, and performed an analysis on the partial averages.

The Monte Carlo data in Table are restricted to rather small sizes $(L \leq 32)$, but have a greater statistical accuracy than other results known to us for such systems. We have analyzed our data according to

$$
g_{L}(K)=g+b L^{y_{i}}+a_{1}\left(K-K_{\mathrm{c}}\right) L^{y_{t}}
$$

with $a_{1}=0.86$ and $y_{t}=1.59[1-5]$, in order to determine $K_{\mathrm{c}}, y_{i}$, and $g$. A least squares fit showed that the data point for $L=4$ deviates significantly, and the fits thus apply to $L \geq 5$.

We found that $g=1.609 \pm 0.004, y_{i}=-0.85 \pm 0.04$, in good agreement with a recent series result of Nickel and Rehr [2]: $y_{i}=-0.83 \pm 0.05$. If we had assumed a fixed value $y_{i}=-0.8$, as was done by Livet [5], we would have obtained $K_{\mathrm{c}}=0.2216445(12)$ for the critical point. Including $y_{i}$ as a variable, our final estimate of the critical point is

$$
K_{\mathrm{c}}=0.221649(4) \text {. }
$$

If the critical point is indeed within these error bounds, Rosengren's conjecture [10] $\tanh K_{\mathrm{c}}=(\sqrt{5}-2) \cos (\pi / 8)$ or $K_{\mathrm{c}} \approx 0.2216586$ is incorrect. Our result for 
$K_{\mathrm{c}}$ is about two standard errors smaller than that of Ferrenberg and Landau [4], and three or more standard errors larger than values listed by Liu and Fisher [1].

TABLE

Finite-size results for the universal ratio $g_{L}$ of the three-dimensional Ising model at a coupling $K=0.221656$. The first column shows the finite size $L$, and the second one our Monte Carlo data with standard errors in the last decimal place between parentheses. The next column shows the length of the simulations. This length expresses the total number of cluster configurations. Most of these were generated by the Swendsen-Wang algorithm, but also are included 5.2, 1.6, 2.0, $1.2,1.2,0.8,0.2,0.2$, and $0.8 \times 10^{7}$ configurations for systems with $L=3,5$, $12,13,14,18,22,24$, and 32 respectively, that were obtained by means of the largest-cluster method. Furthermore, a number of configurations generated by the Wolff algorithm are included: $4 \times 10^{7}$ for $L=3,2 \times 10^{7}$ for $L=4$, and $10^{7}$ for $L=24$ and 28. Five Wolff steps per configuration were used for $L=3$ and 4 , and ten Wolff steps for the larger systems. The fourth column lists our exact numerical results, and the last one contains, for comparison, some numerical results of Ref. [5].

\begin{tabular}{|c|c|c|c|c|}
\hline$L$ & $g_{L}$ (present) & \# conf. & $g_{L}$ (exact) & $g_{L}$ (Ref. [5]) \\
\hline 3 & $1.49599(7)$ & $1.4 \times 10^{8}$ & 1.496016 & \\
\hline 4 & $1.51565(7)$ & $1.8 \times 10^{8}$ & 1.515605 & $1.5167(6)$ \\
\hline 5 & $1.52956(14)$ & $6.4 \times 10^{7}$ & & \\
\hline 6 & $1.54032(19)$ & $4.8 \times 10^{7}$ & & $1.5431(6)$ \\
\hline 7 & $1.5483(2)$ & $4.8 \times 10^{7}$ & & \\
\hline 8 & $1.5545(2)$ & $4.8 \times 10^{7}$ & & $1.5572(9)$ \\
\hline 9 & $1.5597(2)$ & $4.8 \times 10^{7}$ & & \\
\hline 10 & $1.5636(2)$ & $4.8 \times 10^{7}$ & & \\
\hline 11 & $1.5666(2)$ & $4.8 \times 10^{7}$ & & \\
\hline 12 & $1.5696(2)$ & $4.8 \times 10^{7}$ & & $1.5709(14)$ \\
\hline 13 & $1.5722(3)$ & $4.0 \times 10^{7}$ & & \\
\hline 14 & $1.5740(3)$ & $4.0 \times 10^{7}$ & & \\
\hline 15 & $1.5756(4)$ & $2.0 \times 10^{7}$ & & \\
\hline 16 & $1.5766(4)$ & $2.0 \times 10^{7}$ & & $1.5791(13)$ \\
\hline 18 & $1.5790(4)$ & $2.0 \times 10^{7}$ & & \\
\hline 20 & $1.5825(4)$ & $2.0 \times 10^{7}$ & & \\
\hline 22 & $1.5837(4)$ & $2.0 \times 10^{7}$ & & \\
\hline 24 & $1.5857(4)$ & $2.0 \times 10^{7}$ & & $1.5820(25)$ \\
\hline 28 & $1.5867(4)$ & $2.0 \times 10^{7}$ & & \\
\hline 32 & $1.5875(7)$ & $1.0 \times 10^{7}$ & & $1.5943(28)$ \\
\hline
\end{tabular}


In order to gain insight in the discrepancies as mentioned above, it is essential that verifiable numerical data are published, as was done by Livet [5]. Likewise, it is important to specify the details of the random-number generator. This holds especially in the case of the three-dimensional Ising model, where we are confronted with an increasing body of mutually conflicting results.

\section{Acknowledgments}

It is a pleasure to thank J.R. Heringa, A. Kooiman and M. Novotny for valuable discussions.

\section{R.eferences}

[1] A.J. Liu, M.E. Fisher, Physica A 156, 35 (1989).

[2] B.G. Nickel, J.J. Rehr, J. Stat. Phys. 61, 1 (1990).

[3] J.C. Le Guillou, J. Zinn-Justin, Phys. Rev. B 21, 3976 (1980).

[4] A.M. Ferrenberg, D.P. Landau, Phys. Rev. B 44, 5081 (1991).

[5] F. Livet, Europhys. Lett. 16, 139 (1991).

[6] C.F. Baillie, R. Gupta, K.A. Hawick, G.S. Pawley, Phys. Rev. B 45, 10438 (1992).

[7] R.H. Swendsen, J.S. Wang, Phys. Rev. Lett. 58, 86 (1987).

[8] U. Wolff, Phys. Rev. Lett. 60, 1461 (1988).

[9] G. Kamieniarz, H.W.J. Blöte, J. Phys. A 26, 201 (1993).

[10] A. Rosengren, J. Phys. A 19, 1709 (1986). 